Original Research

\title{
Normative Parameters of Gastrocnemius Muscle Stiffness and Associations with Patient Characteristics and Function
}

\author{
Larisa R Hoffman, PT, PhD ${ }^{1}$, Shane L Koppenhaver, PT, PhD², Cameron W MacDonald, $\mathrm{PT}^{1}$, Johnny M Herrera, SPT ${ }^{1}$, \\ Joshua Streuli, SPT ${ }^{1}$, Zachary L Visco, SPT ${ }^{1}$, Nicole Wildermuth, SPT ${ }^{1}$, Stephanie R Albin, PT, PhD ${ }^{1}$ \\ ${ }^{1}$ Regis University, ${ }^{2}$ Baylor University \\ Keywords: stiffness, normative parameters, muscle, gastrocnemius, force production \\ https://doi.org/10.26603/001c.18803
}

International Journal of Sports Physical Therapy

Vol. 16, Issue 1, 2021

\section{Background}

Quantifying muscle stiffness may aid in the diagnosis and management of individuals with muscle pathology. Therefore, the primary purpose of this study was to establish normative parameters and variance estimates of muscle stiffness in the gastrocnemius muscle in a resting and contracted state. A secondary aim was to identify demographic, anthropometric, medical history factors, and biomechanical factors related to muscle stiffness.

\section{Methods}

Stiffness of the gastrocnemius muscle was measured in both a resting and contracted state in 102 asymptomatic individuals in this cross-sectional study. Differences based on muscle state (resting vs contracted) and sex (female vs male) were assessed using a 2 X 2 analysis of variance (ANOVA). Associations between muscle stiffness and sex, age, BMI, race, exercise frequency, exercise duration, force production, and step length were assessed using correlation analysis.

\section{Results}

Gastrocnemius muscle stiffness significantly increased from a resting to a contracted state [mean difference: 217.5 (95\% CI: 191.3, 243.8), $\mathrm{p}<0.001$ ]. In addition, muscles stiffness was $35 \%$ greater for males than females in a resting state and $76 \%$ greater in a contracted state. Greater muscle stiffness in a relaxed and contracted state was associated with larger plantarflexion force production $(r=.26, \mathrm{p}<0.01$ and $r=.23, \mathrm{p}<0.01$ respectively).

\section{Conclusion}

Identifying normative parameters and variance estimates of muscle stiffness in asymptomatic individuals may help guide diagnosing and managing individuals with aberrant muscle function.

\section{Level of Evidence}

2b Individual Cohort Study

\section{Clinical Relevance}

What is known about the subject: Muscle stiffness has been shown to be related to individuals with pathology such as Achilles tendinopathy; however, research is sparse regarding normative values of muscle stiffness. Measuring muscle stiffness may also be a way to potentially predict individuals prone to injury or to monitor the effectiveness of

\footnotetext{
a Corresponding author:

Larisa R. Hoffman,

Regis University,

3333 Regis Blvd, Denver, CO, US.

E-mail:1hoffman@regis.edu
} 
management strategies.

What this study adds to existing knowledge: This study establishes defined estimates of muscle stiffness of the gastrocnemius in both a relaxed and contracted state in healthy individuals. Myotonometry measures of muscle stiffness demonstrated an increase in stiffness during contraction that varies by sex. Greater gastrocnemius muscle stiffness was associated with increased plantarflexion force production.

\section{INTRODUCTION}

Muscle stiffness is related to both passive muscle tension and muscle contraction, and this relationship suggests that muscle stiffness could be used as a surrogate for other measures to estimate changes in muscle force production. ${ }^{1}$ Variances in muscle stiffness have been found to differentiate individuals who have healthy skeletal muscle from individuals who have pathology. Greater muscle stiffness has been observed in individuals with spasticity, ${ }^{2}$ individuals with muscle contracture, ${ }^{2}$ and individuals with muscle pathology. ${ }^{3}$ Other groups of individuals with pathology demonstrate lower levels of muscle stiffness. Lower levels of muscle stiffness have been observed in individuals with Achilles tendinopathy ${ }^{4}$ and individuals who had surgical repair of the Achilles tendon. ${ }^{5}$ The measurement of muscle stiffness may provide an opportunity to compare individuals with healthy muscle performance to individuals with pathology and to potentially monitor effectiveness of management strategies for individuals being managed with a muscle pathology. 1,6,7

Muscle stiffness during contraction is modulated by level of voluntary contraction, joint position, and posture. Muscle stiffness increases dramatically between resting state and contracted state. ${ }^{8}$ Position of the joint also influences the contribution of muscle stiffness. When positioned in ankle dorsiflexion, there is greater muscle stiffness attributed to spinal reflexes; however, when the ankle is positioned into plantarflexion, the amount of stiffness related to spinal reflexes is significantly less. ${ }^{8}$ Further, changing an individual's posture from a prone to a standing position can impact muscle stiffness. The excitability of spinal reflexes (measured through $\mathrm{H}$-wave/M-wave ratios) are dampened in a standing position compared to a prone position. ${ }^{9}$ This difference in positioning demonstrates the value of measuring muscle stiffness in both a resting, prone position, as well as a standing, contracted position.

The primary purpose of this study was to establish normative parameters and variance estimates of muscle stiffness in the gastrocnemius muscle at rest in a prone position and during contraction in a standing posture using the MyotonPRO in a large group of healthy individuals. A secondary aim of this paper is to identify demographic, anthropometric, and medical history factors that are related to muscle stiffness. We hypothesized that lower muscle stiffness would be associated with older age, greater body mass index (BMI), female sex, and the Caucasian race. The third aim of this study was to quantify the relationship between gastrocnemius muscle stiffness and ankle plantarflexion force production and gait parameters. We hypothesized that both resting and contracted muscle stiffness would positively correlate with plantarflexion force, gait velocity, and step length.

\section{METHODS}

\section{PARTICIPANTS}

A total of 102 participants between the ages of 18 to 50 years-of-age were recruited between August 2018 to December 2019. Participants were excluded if they had been treated with dry needling to the lower extremity within the previous 30 days, had a calf injury within the previous six months, were unable to perform a bilateral heel raise symmetrically, were unable to lie on their stomach, or had a previous fracture of the spine or lower extremity that would affect their gait pattern or strength of the gastrocnemius. This study was approved by the Regis University Institutional Review Board and all participants provided informed consent in accordance with the WORLD Medical Association Declaration of Helsinki: Ethical principles for medical research involving human subjects.

\section{PROCEDURES}

This single-group cross-sectional design involved a baseline examination followed by a single measurement session. All measures were performed by examiners specifically trained in all procedures. After providing informed consent, participants were screened for inclusion and exclusion criteria using demographics, medical history questionnaire, and a brief physical examination. The demographics questionnaire included race, sex, previous injuries of the lower extremity, and exercise participation consisting of frequency (greater than 5 days per week, 3-4 days per week, 1-2 days per week, and no regular exercise), duration of session (greater than 60 minutes, 30-60 minutes, and less than 30 minutes), and mode (walking, jogging, cycling, aerobics, swimming, skiing, rock/ice climbing, and hiking). Participants also completed the Beck Anxiety Inventory. Height and weight were measured, and BMI was calculated.

\section{MYOTONOMETRY}

All myotonometry was performed using the MyotonPRO (Myoton AS, Tallinn, Estonia) to assess mechanical stiffness of the medial head of the gastrocnemius muscle. Tissue stiffness (elasticity) is most commonly quantified as Young's modulus, which is defined as the slope of the stress-strain curve of a material in the elastic deformation region of interest. ${ }^{10}$ The MyotonPRO applies a mechanical impulse to the skin which is then transmitted to the underlying soft tissue and muscle ( $0.58 \mathrm{~N}$ for 15 milliseconds). ${ }^{11}$ This mechanical impulse causes the muscle to respond by a dampened natural oscillation, which is recorded by an accelerometer in the form of an acceleration signal. This acceleration signal is then utilized to calculate Young's modulus and other viscoelastic parameters. The oscillation of the muscles is recorded by the probe in order to calculate 
the mechanical stiffness $(\mathrm{N} / \mathrm{m})$ of the muscle. ${ }^{12}$ A standard site was marked on each participant four fingerbreadths below the popliteal crease in the belly of the medial gastrocnemius muscle. ${ }^{13}$ All measures were taken at this standardized site. Participants were measured in a resting and contracted state. For resting state measures, participants were positioned in prone with shoes and socks removed and feet unsupported in a resting position off the end of the table and knees in full extension. For contracted state measures, participants stood with a scale under each foot and asked to place equal weight between their right and left feet. They were asked to raise onto their toes (perform a bilateral heel raise) as high as they could and hold the position while the measures were taken (Figure 1). As individuals performed the heel raise position, an assessor made sure equal weight was maintained between the lower extremities as well as consistent heel height between trials.

Three measures were taken in each position and averaged. Reliability of the MytonPRO in a resting state has been shown to be 0.99 to $1.0^{13}$ and in a contracted state in a standing position within day ICC was 0.94 and between day ICC was 0.99.14

\section{PLANTARFLEXION FORCE PRODUCTION MEASUREMENT}

Force production of the gastrocnemius muscles was assessed with a hand-held dynamometer (HHD) (Hoggan Scientific LLC; Salt Lake City, UT, USA). The HHD was anchored to the wall. The participant was strapped to the table (Figure 2) with a strap across the popliteal crease and across the pelvis as previously described by Kelly et al. ${ }^{13}$ The ankle was positioned to maintain 0 degrees of ankle plantarflexion for the isometric contraction. The dynamometer pad was placed at the first metatarsal head and the participant was asked to push with maximal force. An average of 3 trials was calculated.

\section{GAIT ANALYSIS}

Gait analysis was performed using the GAITRite system (CIR Systems, Inc. Sparta, NJ, USA). The 6-meter measurement area of the mat is $61 \mathrm{~cm}$ wide and $488 \mathrm{~cm}$ long. The sensors are arranged in a grid pattern $(48 \mathrm{~cm} \mathrm{X} 384 \mathrm{~cm})$ and placed $1.27 \mathrm{~cm}$ from apart. Sampling rate of the system varies between 32.2 and 38.4 Hz. Participants walked across the mat barefoot and an average of 3 trials was used for analysis. All data were automatically uploaded to a computer as the participant walked across the mat. Spatial and temporal characteristics of gait included normalized gait velocity and step length. ${ }^{15}$

\section{DATA ANALYSIS}

All statistical analyses were performed using SPSS 26.0 software (IBM SPSS Inc, Armonk, NY, USA). Descriptive statistics were calculated for all demographic, anthropometric, and medical history variables. Mechanical stiffness of the gastrocnemius muscle was estimated for the cohort and separately for male and female participants. Based on 1,000 bootstrap samples, 95\% confidence intervals were estimated. Statistical comparisons of muscle stiffness were

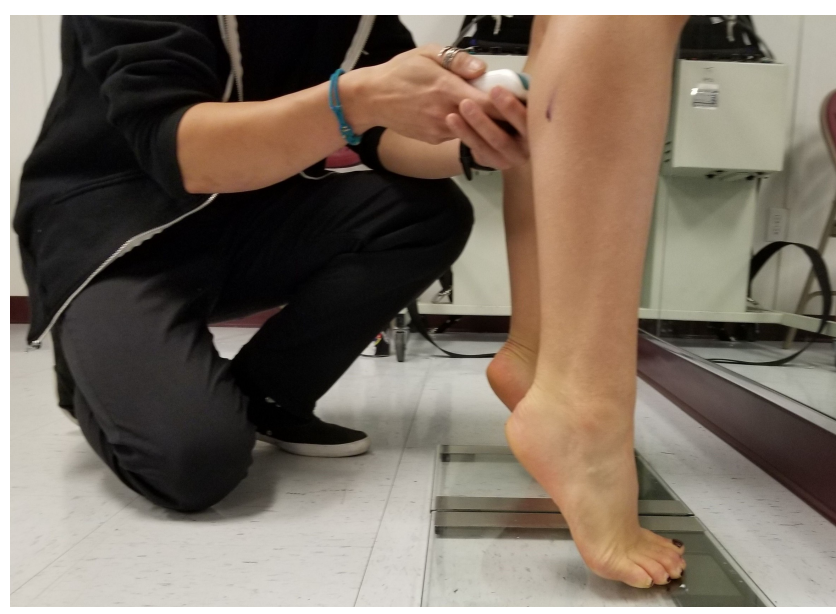

Figure 1: Muscle Stiffness Measured in a Contracted State

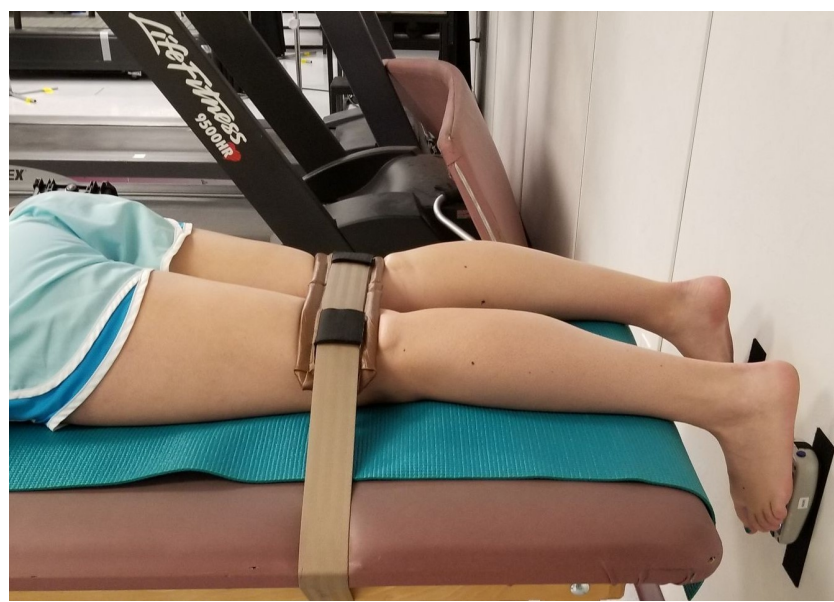

Figure 2: Plantarflexion Force Production Measured in a Prone Position

made between the muscle condition (resting state and contracted state) and sex (male and female) using a 2 X 2 analysis of variance (ANOVA). Findings were considered statistically significant when $\mathrm{p}<0.05$.

The associations between muscle stiffness and the following demographic and anthropometric variables were analyzed based on theoretical plausibility: sex, age, BMI, race, exercise frequency, and exercise duration. Bivariate associations between muscle stiffness and each demographic and anthropometric variable were assessed using correlation coefficients (point biserial for dichotomous variables, spearman rho for ordinal variables, and Pearson product moment for continuous variables). Similarly, bivariate associations between muscle stiffness, force production, and gait parameters (normalized velocity and step length) were assessed using Pearson product moment coefficients. All correlation coefficients included $95 \%$ confidence intervals estimated based on 1,000 bootstrap samples and all associations were considered statistically significant if $\mathrm{p}<0.05$. 
Table 1: Demographics of Participants

\begin{tabular}{ll}
\hline Demographics (N=102) & Descriptive Statistic \\
\hline Age (Mean, Standard Deviation) & $26.00(4.43)$ \\
Sex - Male (n, \%) & $44(42.3 \%)$ \\
Race (n, \%) & \\
$\quad$ Native American & $1(1 \%)$ \\
Asian & $16(15.4 \%)$ \\
African American & $0(0 \%)$ \\
Caucasian & $75(72.1 \%)$ \\
$\quad$ Hispanic & $10(9.6 \%)$ \\
Beck Anxiety Inventory (Mean, Standard Deviation) & $4.34(5.11)$ \\
BMI (Mean, Standard Deviation) & $23.51(3.92)$ \\
\hline
\end{tabular}

Table 2: Normative Parameters in Muscle Stiffness of the Gastrocnemius for All Participants and By Sex in Newton/Meters

\begin{tabular}{|c|c|c|}
\hline & Mean $(95 \% \mathrm{CI})$ & Standard deviation $(95 \% \mathrm{Cl})$ \\
\hline \multicolumn{3}{|l|}{ All participants $(n=102)$} \\
\hline Resting state & $300.1(286.6,312.3)$ & $68.7(56.8,79.2)$ \\
\hline Contracted state & $504.6(461.8,545.1)$ & $208.4(179.7,231.1)$ \\
\hline \multicolumn{3}{|l|}{ Female $(n=58)$} \\
\hline Resting state & $261.95(250.1,271.2)$ & $43.4(34.0,50.1)$ \\
\hline Contracted state & $384.8(347.5,408.7)$ & $132.83(96.2,145.4)$ \\
\hline \multicolumn{3}{|l|}{ Male $(n=44)$} \\
\hline Resting state & $350.3(331.9,370.9)$ & $63.6(49.9,76.0)$ \\
\hline Contracted state & $662.6(609.7,781.7)$ & $183.7(151.1,209.9)$ \\
\hline
\end{tabular}

\section{RESULTS}

One hundred and two participants were recruited between August 2018 and December 2019. No participants that were screened were excluded due to inclusion/exclusion criteria. Demographic, anthropometric, and medical history characteristics of the participants are summarized in Table $\mathbf{1}$.

There was a significant interaction for sex and muscle condition ( $\mathrm{p}<0.001)$; therefore, gastrocnemius muscle stiffness is summarized for both the resting and contracted states as well as separately by sex in Table 2 . Muscle stiffness of the gastrocnemius was significantly higher for males than females [183.1 (95\% CI: 144.8, 221.4), $\mathrm{p}<0.001)]$ and significantly higher in the contracted state than the resting state [217.5 (95\% CI: 191.3, 243.8), p < 0.001].

Association between muscle stiffness and demographic, anthropometric, and medical history characteristics are presented in Table 3 . There were significant associations for sex and muscle stiffness in both a resting state and contracted state. In a contracted state, muscle stiffness was also associated with anxiety. Sex was associated with muscle stiffness in both states with males exhibiting greater muscle stiffness (see Table 2). A significant negative association was found between muscle stiffness in a contracted state and anxiety suggesting that individuals that reported higher levels of anxiety, as measured by the Beck Anxiety Inventory, had lower levels of muscle stiffness in a contracted state.

Associations between gastrocnemius muscle stiffness in resting and contracted states were significant with muscle force production of the gastoc-soleus complex (see Table 4). The association of muscle stiffness in both a resting and contracted state was positive, indicating that higher muscle stiffness was associated with greater force production. A positive association was also found for step length and muscle stiffness in both states, indicating that individuals with higher muscle stiffness exhibit longer step length. Age, BMI, race, exercise frequency, exercise duration, and normalized gait velocity were not associated with gastrocnemius muscle stiffness in a resting or contracted state.

\section{DISCUSSION}

The purpose of this study was to establish normative parameters and variance estimates of muscle stiffness in the gastrocnemius muscle in a resting and contracted state using the myotonometry in a large group of healthy individuals. The confidence intervals for muscle stiffness in a rest- 
Table 3: Association of Gastrocnemius Muscle Stiffness with Demographic, Anthropometric, and History Variables

\begin{tabular}{lll}
\hline & Resting State $(95 \% \mathrm{CI})$ & Contracted State $(95 \% \mathrm{Cl})$ \\
\hline Sex & $-.606(-.719,-.480)^{* *}$ & $-.613(-.757,-.437)^{* *}$ \\
Age & $-.023(-.166, .155)$ & $.069(-.091, .259)$ \\
BMI & $-.027(-.224, .262)$ & $-.102(-.245, .110)$ \\
Beck Anxiety Inventory & $-.180(-.312,-.032)$ & $-.223(-.381,-.027)^{*}$ \\
Race & $-.085(-.274, .132)$ & $-.153(-.349, .086)$ \\
Exercise Frequency & $.076(-.138, .281)$ & $.011(-.219, .230)$ \\
Exercise Duration & $.062(-.152, .286)$ & $.123(-.100, .333)$ \\
\hline
\end{tabular}

Notes: Sex (female =1, male = 0); Race $($ Native-American = 1, Asian = 2, African American = 3, Caucasian = 4, Hispanic $=5$ ).

$* \mathrm{p}<0.05$.

$* * \mathrm{p}<0.01$.

Table 4: Association of Gastrocnemius Muscle Stiffness with Functional Variables

\begin{tabular}{lll}
\hline & Resting State $(95 \% \mathrm{Cl})$ & Contracted State $(95 \% \mathrm{Cl})$ \\
\hline Plantarflexion Force & $.264(.026, .461)^{* *}$ & $.232(.062, .387)^{* *}$ \\
Normalized Gait Velocity & $-.066(-.247, .130)$ & $.050(-.138, .247)$ \\
Step Length & $.230(.029, .426)^{*}$ & $.288(.112, .448)^{* *}$ \\
\hline
\end{tabular}

$* \mathrm{p}<0.05$.

** $\mathrm{p}<0.01$.

ing and contracted state were small (Table 2), suggesting that the estimates are fairly precise. Muscle stiffness in a resting state was 300.07 , whereas it was 504.62 in a contracted state, which is $68 \%$ higher than the resting state. This likely represents the amount of "stiffening" due to submaximal muscle contraction and is consistent with the findings of other investigators which have also observed greater muscle stiffness in a contracted state. ${ }^{13}$

Muscle stiffness of the gastrocnemius in both a relaxed and contracted state was significantly higher for males than females. In this study, muscle stiffness, as measured by myotonometry, demonstrated males had 35\% higher muscle stiffness than females, and in a contracted state, males had $76 \%$ higher muscle stiffness than females. This is consistent with the findings from other researchers who have investigated sex differences in muscle stiffness in lower extremity muscles. ${ }^{16-20}$ Some investigators have found that sex differences in muscle stiffness are only observed when the muscle is in a tensioned state (such as a position of stretch on the gastrocnemius in a resting dorsiflexed position); ${ }^{19}$ however, we observed these differences when the muscle was on slack (a resting state in approximately 20 degrees of plantarflexion) and under tension (a contracted state in a plantarflexed position). Interestingly, other investigators have found muscle stiffness (measured using shear wave elastography) was higher in females than males. ${ }^{21,22}$ Both of these studies investigated stiffness in the biceps muscle. It is possible that muscle stiffness is muscle or region specific. Based on findings from this study, future studies utilizing myotonometry could consider sex as a potential co- variate.

A secondary aim of this paper was to identify demographic, anthropometric, and medical history factors related to muscle stiffness. Muscle stiffness was negatively correlated with anxiety. This was not surprising, as the relationship between anxiety and musculoskeletal pain has been documented. ${ }^{23}$ The relationship between anxiety and muscle stiffness is important to note, as anxiety maybe a confounding variable influencing muscle stiffness during recovery. We also observed increased anxiety in females more than males. Other investigators have also observed that females maybe more vulnerable to anxiety related to musculoskeletal pain. 24,25

Interestingly, in this study we did not find significant relationships between gastrocnemius muscle stiffness and age, BMI, ethnicity, exercise frequency, exercise duration, or gait velocity. Other investigators who have only included young and middle-aged adults have also found no correlation between stiffness and age. ${ }^{26}$ Studies that included older age groups (with an age range of 24-94 years of age) have observed a decrease in muscle stiffness with increasing age. ${ }^{27}$ In this study, we excluded older individuals (above the age of 65-years-old). The age range in this study was 24-45 years of age.

We did not observe a relationship between BMI and muscle stiffness. Investigations of the relationship between BMI and muscle stiffness in neck muscles only found a correlation in one muscle group out of multiple neck muscles measured. ${ }^{28}$ Both this study and the investigation by Kuo et al. included only individuals classified as normal or un- 
derweight. Future research should include individuals classified in the overweight or obese categories to compare to the muscle stiffness in individuals who are classified as normal weight.

We did not observe a relationship between race and muscle stiffness, but it is likely that the lack of diversity in this sample (73.5\% being Caucasian) may have influenced this finding. Previous research has indicated that muscle stiffness is greater amongst athletes whom are African American. ${ }^{29}$

We did not find a significant relationship between muscle stiffness and exercise frequency or duration. This finding was in contrast to previous research. Other investigators have found that exercise is associated with increases in muscle stiffness. ${ }^{30,31}$ We divided exercise frequency and duration into four different categories. Exercise frequency consisted of the following categories: greater than 5 days per week, 3 to 4 days per week, 1 to 2 days per week, and no regular exercise. We divided exercise duration into the following 3 categories: less than 30 minutes, 30-60 minutes, and greater than 60 minutes. It is possible that the ordinal scale of exercise participation used in this study is not sensitive enough to distinguish differences between groups.

The third aim of this study was to determine if muscle stiffness influences mechanical factors, such as muscle force production and gait parameters. Muscle stiffness in both a relaxed and contracted state was found to be significantly correlated with force production of the gastrocsoleus complex and step length during walking. Some investigators have observed a relationship between muscle force production in the tibialis anterior and muscle stiffness (measured with shear wave elastography), ${ }^{32}$ while others have only found a correlation between rapid force production and muscle stiffness. ${ }^{33}$ This relationship between muscle performance and muscle stiffness indicates that muscle stiffness could be a valid, alternative measure to examine muscle performance. ${ }^{1}$

Ankle muscle stiffness varies during walking according to the phase of gait, with greater muscle stiffness occurring during terminal stance and pre-swing phases of gait. ${ }^{34}$ Therefore, theoretically, individuals with greater muscle stiffness will be able to maintain late stance phases of gait for a longer time and thereby increase step length, as we observed in this study. However, aberrant muscle stiffness, either excessive or insufficient muscle stiffness, may change this relationship. Excessive muscle stiffness (observed in children with spastic cerebral palsy) interferes with the ability to achieve the second and third ankle rockers. ${ }^{34} \mathrm{Al}-$ ternatively, insufficient muscle stiffness (observed in adults with peripheral neuropathy) could also interfere with efficiency of motion during these late stance phases of gait, leading to reduced gait speed. ${ }^{35}$ Further supporting this idea, manipulating muscle stiffness through tibial nerve block is associated with a reduction in stride length. ${ }^{36}$

We did not observe a relationship between gait velocity and muscle stiffness, whereas other investigators have observed a relationship between these two parameters. Increased muscle stiffness has been shown to be related to increased running economy, ${ }^{37}$ and reduced muscle stiffness has been shown to be a factor influencing reduced gait speed. ${ }^{35}$ For individuals with peripheral neuropathy, plantarflexor torque explained most of the variance in gait speed, but plantarflexor muscle stiffness also contributed. 35 In this study, we excluded individuals with gait deviations, which may explain the lack of a relationship between gait speed and muscle stiffness.

Limitations. This study has several limitations which should be recognized. The homogenous sample may have impacted the results, specifically the lack of a relationship between muscle stiffness and age, race, BMI, gait velocity, and exercise frequency and duration. The ordinal scale designed to measure exercise frequency and duration may not be a valid measure of activity level. Finally, this study excluded individuals with lower extremity injury, which limits the application to individuals with musculoskeletal impairments. Future studies should include a greater diversity of participants, including individuals with musculoskeletal impairments.

\section{CONCLUSION}

This study established defined estimates of muscle stiffness of the gastrocnemius in both a relaxed and contracted state in healthy individuals. Myotonometry measures of muscle stiffness demonstrated an increase in stiffness during contraction that varies by sex. Greater gastrocnemius muscle stiffness was associated with increased plantarflexion force production. Establishing estimates of muscle stiffness in healthy individuals may aid in identifying individuals with aberrant muscle stiffness who may be prone to injury, variations in normal muscular function and inform goal setting in rehabilitation.

\section{FINANCIAL DISCLOSURE}

We affirm that we have no financial affiliation or involvement with any commercial organization that has a direct financial interest in any matter included in this manuscript, except as cited in the manuscript.

\section{CONFLICTS OF INTEREST}

All authors state declaration of interest: none.

Submitted: June 06, 2020 CDT, Accepted: October 09, 2020 CDT 


\section{REFERENCES}

1. Hug F, Tucker K, Gennisson J-L, Tanter M, Nordez A. Elastography for Muscle Biomechanics: Toward the Estimation of Individual Muscle Force. Exerc Sport Sci Rev. 2015;43(3):125-133. doi:10.1249/jes.0000000000 $\underline{000049}$

2. Kwon DR, Park GY, Lee SU, Chung I. Spastic cerebral palsy in children: Dynamic sonoelastographic findings of medial gastrocnemius. Radiology. 2012;263(3):794-801. doi:10.1148/radiol.12 102478

3. Drakonaki EE, Allen GM. Magnetic resonance imaging, ultrasound and real-time ultrasound elastography of the thigh muscles in congenital muscle dystrophy. Skeletal Radiol. 2010;39(4):391-396. doi:10.1007/s00256-009-0861-0

4. Morgan G, Martin R, Welch H, Williams L, Morris K. Objective assessment of stiffness in the gastrocnemius muscle in patients with symptomatic Achilles tendons. BMJ Open Sport Exerc Med. 2019;5(1):e000622. doi:10.1136/bmjsem-2019-000622

5. Borges PRT, Santos TRT, Procópio PRS, Chelidonopoulos JHD, Zambelli R, Ocarino JM. Passive stiffness of the ankle and plantar flexor muscle performance after Achilles tendon repair: A cross-sectional study. Braz J Phys Ther. 2017;21(1):51-57. doi:10.1016/i.bjpt.2016.12.004

6. Brandenburg JE, Eby SF, Song P, et al. Ultrasound elastography: The new frontier in direct measurement of muscle stiffness. Arch Phys Med Rehabil. 2014;95(11):2207-2219. doi:10.1016/j.apmr.2 $\underline{014.07 .007}$

7. Butler RJ, Crowell HPI, Davis IM. Lower extremity stiffness: Implications for performance and injury. Clin Biomech. 2003;18(6):511-517. doi:10.1016/s026 8-0033(03)00071-8

8. Mirbagheri MM, Barbeau H, Kearney RE. Intrinsic and reflex contributions to human ankle stiffness: Variation with activation level and position. Exp Brain Res. 2000;135(4):423-436. doi:10.1007/s00221000053 $\underline{4}$

9. Koceja DM, Trimble MH, Earles DR. Inhibition of the soleus H-reflex in standing man. Brain Res. 1993;629(1):155-158. doi:10.1016/0006-8993(93)9049 $\underline{5-9}$

10. Gennisson JL, Deffieux T, Mace E, et al. Viscoelastic and anisotropic mechanical properties of in vivo muscle tissue assessed by supersonic shear imaging. Ultrasound Med Biol. 2010;36(5):789-801.
11. Ditroilo M, Hunter AM, Haslam S, De Vito G. The effectiveness of two novel techniques in establishing the mechanical and contractile responses of biceps femoris. Physiol Meas. 2011;32(8):1315-1326. doi:10.1 $\underline{088 / 0967-3334 / 32 / 8 / 020}$

12. Nair K, Masi AT, Andonian BJ, et al. Stiffness of resting lumbar myofascia in healthy young subjects quantified using a handheld myotonometer and concurrently with surface electromyography monitoring. J Bodyw Mov Ther. 2016;20(2):388-396. d oi:10.1016/j.jbmt.2015.12.005

13. Kelly JP, Koppenhaver SL, Michener LA, Proulx L, Bisagni F, Cleland JA. Characterization of tissue stiffness of the infraspinatus, erector spinae, and gastrocnemius muscle using ultrasound shear wave elastography and superficial mechanical deformation. J Electromyogr Kinesiol. 2018;38:73-80. doi:10.1016/j.j elekin.2017.11.001

14. Albin SR, Koppenhaver SL, Bailey B, et al. The effect of manual therapy on gastrocnemius muscle stiffness in healthy individuals. Foot. 2019;38:70-75. doi:10.1016/j.foot.2019.01.006

15. van Uden CJ, Besser MP. Test-retest reliability of temporal and spatial gait characteristics measured with an instrumented walkway system (GAITRite ${ }^{\circledR}$ ). BMC Musculoskelet Disord. 2004;5(1):13. doi:10.1186/ 1471-2474-5-13

16. Blackburn JT, Padua DA, Weinhold PS, Guskiewicz KM. Comparison of triceps surae structural stiffness and material modulus across sex. Clinical Biomechanics. 2006;21(2):159-167. doi:10.1016/j.clinb iomech.2005.08.012

17. Blackburn JT, Riemann BL, Padua DA, Guskiewicz KM. Sex comparison of extensibility, passive, and active stiffness of the knee flexors. Clinical Biomechanics. 2004;19(1):36-43. doi:10.1016/i.clinbio mech.2003.09.003

18. Blackburn JT, Bell DR, Norcross MF, Hudson JD, Kimsey MH. Sex comparison of hamstring structural and material properties. Clin Biomech. 2009;24(1):65-70. doi:10.1016/i.clinbiomech.2008.1 $\underline{0.001}$

19. Miyamoto N, Hirata K, Miyamoto-Mikami E, Yasuda O, Kanehisa H. Associations of passive muscle stiffness, muscle stretch tolerance, and muscle slack angle with range of motion: Individual and sex differences. Sci Rep. 2018;8(1):8274. doi:10.1038/s415 98-018-26574-3 
20. Riemann BL, DeMont RG, Ryu K, et al. The Effects of Sex, Joint Angle, and the Gastrocnemius Muscle on Passive Ankle Joint Complex Stiffness. J Athl Train. $2001 ; 36(4): 369-375$.

21. Chen J, O’Dell M, He W, Du L-J, Li P-C, Gao J. Ultrasound shear wave elastography in the assessment of passive biceps brachii muscle stiffness: Influences of sex and elbow position. Clin Imaging. 2017;45:26-29. doi:10.1016/j.clinimag.2017.05.017

22. Eby SF, Cloud BA, Brandenburg JE, et al. Shear wave elastography of passive skeletal muscle stiffness: Influences of sex and age throughout adulthood. Clinical Biomech. 2015;30(1):22-27. doi:1 0.1016/j.clinbiomech.2014.11.011

23. Lucchetti G, Oliveira AB, Mercante JPP, Peres MFP. Anxiety and fear-avoidance in musculoskeletal pain. Curr Pain Headache Rep. 2012;16(5):399-406. doi:10.1 007/s11916-012-0286-7

24. Bingefors K, Isacson D. Epidemiology, comorbidity, and impact on health-related quality of life of self-reported headache and musculoskeletal pain-a gender perspective. Eur J Pain. 2004;8(5):435-450. doi:10.1016/j.ejpain.2004.01.005

25. Stoyanova M, Hope DA. Gender, gender roles, and anxiety: Perceived confirmability of self report, behavioral avoidance, and physiological reactivity. $J$ Anxiety Disord. 2012;26(1):206-214. doi:10.1016/i.jan $\underline{\text { xdis.2011.11.006 }}$

26. König M, Hemmers S, Epro G, et al. Matching Participants for Triceps Surae Muscle Strength and Tendon Stiffness Does Not Eliminate Age-Related Differences in Mechanical Power Output During Jumping. Front Physiol. 2018;9:1345. doi:10.3389/fphy s.2018.01345

27. Alfuraih AM, Tan AL, O’Connor P, Emery P, Wakefield RJ. The effect of ageing on shear wave elastography muscle stiffness in adults. Aging Clin Exp Res. 2019;31(12):1755-1763. doi:10.1007/s4052 0-019-01139-0

28. Kuo W-H, Jian D-W, Wang T-G, Wang Y-C. Neck muscle stiffness quantified by sonoelastography is correlated with body mass index and chronic neck pain symptoms. Ultrasound Med Biol. 2013;39(8):1356-1361. doi:10.1016/j.ultrasmedbio.20 12.11.015
29. Fukashiro S, Abe T, Shibayama A, et al. Comparison of viscoelastic characteristics in triceps surae between black and white athletes. Acta Physiol Scand. 2002;175(3):183-187.

30. Hoang PD, Herbert RD, Gandevia SC. Effects of eccentric exercise on passive mechanical properties of human gastrocnemius in vivo. Med Sci Sport Exer. 2007;39(5):849-857. doi:10.1249/mss.0b013e3180334 $\underline{99 \mathrm{~b}}$

31. Lacourpaille L, Nordez A, Hug F, Couturier A, Dibie C, Guilhem G. Time-course effect of exerciseinduced muscle damage on localized muscle mechanical properties assessed using elastography. Acta Physiol (Oxf). 2014;211(1):135-146. doi:10.1111/ apha.12272

32. Sasaki K, Toyama S, Ishii N. Length-force characteristics of in vivo human muscle reflected by supersonic shear imaging. I Appl Physiol. 2014;117(2):153-162. doi:10.1152/japplphysiol.0105 $\underline{8.2013}$

33. Ando R, Suzuki Y. Positive relationship between passive muscle stiffness and rapid force production. Hum Movement Sci. 2019;66:285-291. doi:10.1016/j.hu mov.2019.05.002

34. Davis RB, DeLuca PA. Gait characterization via dynamic joint stiffness. Gait Posture. 1996;4(3):224-231. doi:10.1016/0966-6362(95)0104 $\underline{5-9}$

35. Salsich GB, Mueller MJ. Effect of plantar flexor muscle stiffness on selected gait characteristics. Gait \& Posture. 2000;11(3):207-216. doi:10.1016/s0966-63 62(00)00047-3

36. Farris DJ, Kelly LA, Cresswell AG, Lichtwark GA. The functional importance of human foot muscles for bipedal locomotion. Proc Natl Acad Sci USA. 2019;116(5):1645-1650. doi:10.1073/pnas.181282011 $\underline{6}$

37. Dumke CL, Pfaffenroth CM, McBride JM, McCauley GO. Relationship between muscle strength, power and stiffness and running economy in trained male runners. Int J Sports Physiol Perform. 2010;5(2):249-261. doi:10.1123/ijspp.5.2.249 